\title{
Development of Highly Selective Pyrimidine-Based Aldosterone Synthase (CYP11B2) Inhibitors
}

\author{
Steven M. Sparks, ${ }^{*}{ }^{\dagger}$ ○ Dana P. Danger, ${ }^{\dagger}$ William J. Hoekstra, ${ }^{\dagger}$ Tony Leesnitzer, ${ }^{\dagger}$ Robert J. Schotzinger, ${ }^{\dagger}$
} Christopher M. Yates, ${ }^{\dagger}$ and J. David Becherer ${ }^{\dagger}$

${ }^{\dagger}$ Selenity Therapeutics, 4505 Emperor Boulevard, Durham, North Carolina 27703, United States

${ }^{\ddagger}$ OpAns, 4134 South Alston Avenue, Durham, North Carolina 27713, United States

Supporting Information

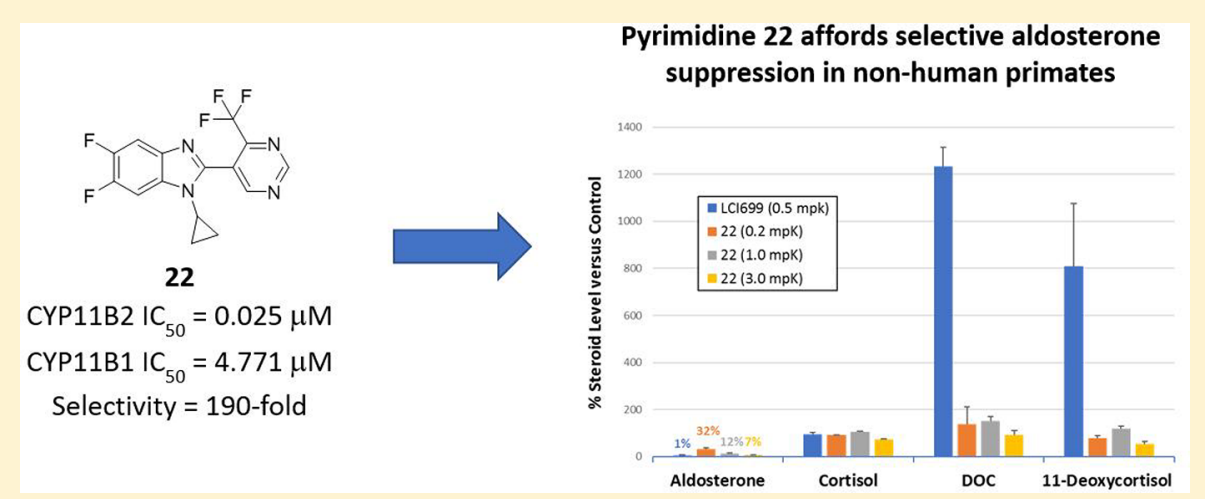

ABSTRACT: Excess aldosterone production and signaling are primary contributors to numerous cardiovascular disorders including primary aldosteronism and resistant hypertension. Recently, inhibition of aldosterone synthesis via the enzyme aldosterone synthase (CYP11B2) has been pursued to ameliorate the negative effects of elevated aldosterone. Herein, we report the development of aldosterone synthase inhibitors using a pyrimidine-based metal binding group leading to the highly selective CYP11B2 inhibitor 22. Superior selectivity combined with robust pharmacokinetics afforded highly selective in vivo aldosterone suppression in a monkey model of adrenal steroidogenesis, demonstrating the potential for selective aldosterone lowering in humans with pyrimidine 22.

KEYWORDS: Aldosterone, cytochrome P450, CYP11B2, CYP11B2 inhibitor

$\mathrm{C}$ hronically elevated aldosterone levels negatively impact the cardiovascular and renal systems through multiple mechanisms including increased blood pressure and promotion of fibrosis and inflammation. ${ }^{1}$ Hyperaldosteronism is also associated with insulin resistance and therefore contributes to the progression of metabolic syndrome. ${ }^{2,3}$ Consequently, patients with elevated aldosterone are at greater risk for serious cardiovascular and renal disorders including heart failure, ischemic heart disease, arrhythmias, stroke, and kidney disease. $^{4-7}$ Due to the negative consequences associated with excess aldosterone levels, interest in inhibiting the production of aldosterone has recently gained attention.

Aldosterone synthase (CYP11B2) is the enzyme located in the zona glomerulosa of the adrenal glands, which is responsible for the production of aldosterone. ${ }^{8}$ Typically under the control of the primary regulators potassium, angiotensin II, and renin, aldosterone elicits its fluid balance effects in the kidney where it activates the mineralocorticoid receptor (MR) leading to sodium and water reabsorption. These effects increase plasma volume resulting in increased blood pressure. Given this sequence, excess aldosterone inappropriately activates $\mathrm{MR}$ contributing to high blood pressure.

CYP11B2 produces aldosterone through a three-step sequence involving sequential oxidations carried out by the heme-iron cofactor of the enzyme. ${ }^{8}$ Starting with 11 deoxycorticosterone (DOC), initial hydroxylation at C-11 affords corticosterone which is hydroxylated at C-18 to furnish 18-hydroxycorticosterone. 18-Hydroxycorticosterone then undergoes a final C-18 oxidation (alcohol to aldehyde) to provide aldosterone. Importantly, CYP11B2 is the only enzyme which catalyzes the final oxidation leading to aldosterone.

A significant obstacle to the development of CYP11B2 inhibitors has been the identification of compounds which selectivity inhibit CYP11B2 over CYP11B1, the enzyme responsible for cortisol production. ${ }^{9}$ The serious complications associated with dysregulated cortisol production, including adrenal insufficiency and adrenal crisis, make inhibition of

Received: April 3, 2019

Accepted: June 6, 2019

Published: June 7, 2019 
CYP11B1 a serious liability that needs to be avoided. Indeed, the development of the first CYP11B2 inhibitor to reach the clinic, LCI699, suffered from poor selectivity against CYP11B1 and was eventually discontinued for the treatment of hypertension for this reason. ${ }^{10,11}$ Given the high homology between the two enzymes (93\%) it is not surprising this challenge exists. ${ }^{8}$ However, several recent reports detail significant progress in the development of selective inhibitors, $^{12-15}$ including the identification of highly selective clinical candidates LFF269 and RO-6836191. ${ }^{16,17}$

Our approach to the design of highly selective metalloenzyme inhibitors focuses on the optimization of the inhibitor metal binding group (MBG)-enzyme metal ion interaction and has been successfully employed for the development of highly selective inhibitors of related cytochrome P450 enzymes, fungal CYP51 and CYP17. ${ }^{18,19}$ Recognition that overly potent MBGs (pyridines and imidazoles for heme-iron) exert potent off-target activities causing side-effects, led to consideration of less metal-avid MBGs to improve selectivity, and provide an opportunity for an improved therapeutic window. ${ }^{20}$ As this strategy typically identifies less potent inhibitors with improved selectivity, modifications to the rest of the scaffold are utilized to enhance potency while retaining the high selectivity imparted by the optimized MBG. Given our success employing this strategy for related cytochrome P450s, application to CYP11B2 was undertaken.

A survey of the CYP11B2 literature revealed several screening hits utilizing pyridine and imidazole MBGs affording modest selectivity which were subsequently developed into selective inhibitors. Given the favorable developability properties, the simple 2-(3-pyridyl) indole and benzimidazole screening hits 1 and $\mathbf{2}$ were selected for further evaluation (Figure 1). ${ }^{12,16}$

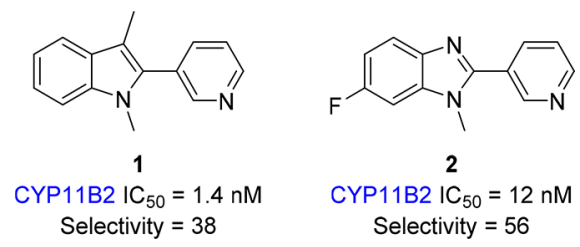

Figure 1. Indole and benzimidazole based CYP11B2 scaffolds.

Facile synthetic access to the benzimidazole scaffold allowed for efficient preparation of a series of inhibitors with varied MBGs (Table 1). ${ }^{21}$ Following precedent, the benzimidazole core was adorned with N1 cyclopropyl and 6-fluoro substituents for the MBG scan with published data for pyridine-containing inhibitor $\mathbf{1 1}$ included for comparison. ${ }^{12}$ Compounds were tested for inhibition of human recombinant CYP11B2 or CYP11B1 utilizing an HPLC-MS readout to monitor both substrate and product steroids. ${ }^{22}$

A variety of five- and six-membered ring nitrogen-containing heterocyclic MBGs were evaluated with positions adjacent to the nitrogen atom unencumbered to allow for effective interaction with the heme-iron. In general, six-membered ring heterocycles were preferred over five-membered heterocycles (7-10 vs 3-5) with a single five-membered heterocycle, thiazole 6, affording good potency and encouraging selectivity. Intrigued by the weak CYP11B1 activity and high selectivity afforded by the pyrimidine analogue 7 , further exploration was undertaken. ${ }^{23}$
Table 1. MBG Evaluation of Benzimidazole Scaffold 2

\begin{tabular}{|c|c|c|c|c|}
\hline cmpd & MBG & $\underset{(\mu \mathrm{M})}{\mathrm{CYP} 11 \mathrm{~B} 2 \mathrm{IC}_{50}}$ & $\underset{(\mu \mathrm{M})}{\mathrm{CYP}^{11 \mathrm{~B} 1 \mathrm{IC}_{50}}}$ & selectivity \\
\hline 3 & $\begin{array}{r}1-1,2,3- \\
\text { triazole }\end{array}$ & 1.064 & 9.072 & 8.5 \\
\hline 4 & 5-oxazole & $>10.0$ & $>10.0$ & N.A. \\
\hline 5 & 4-pyrazole & 0.782 & 1.668 & 2 \\
\hline 6 & 5-thiazole & 0.062 & 4.523 & 73 \\
\hline 7 & 5-pyrimidine & 0.040 & 5.380 & 136 \\
\hline 8 & 2-pyrazine & 0.541 & $>10.0$ & $>18$ \\
\hline 9 & 3-pyridazine & 0.412 & $>10.0$ & $>24$ \\
\hline 10 & 4-pyridazine & 0.330 & $>10.0$ & $>30$ \\
\hline $11^{12}$ & 3-pyridine & 0.004 & 0.401 & 100 \\
\hline
\end{tabular}

Examination of simple substitution of the pyrimidine is shown in Table 2 utilizing the 5,6-difluorobenzimidazole scaffold. As expected, simple 2-methyl substitution on the pyrimidine ring provided compounds devoid of CYP11B2 activity (data not shown), likely due to disruption of the pyrimidine nitrogen-heme iron interaction. In addition, 4,6dimethyl substitution was also inactive (data not shown), potentially suggesting some degree of coplanarity between the MBG and benzimidazole core is required for binding in the CYP11B2 pocket. Increasing the size of simple alkyl substituents at $\mathrm{C} 4(\mathbf{1 2} \rightarrow \mathbf{1 3} \rightarrow \mathbf{1 4} \rightarrow \mathbf{1 5})$ led to increased potency at CYP11B2 with compounds 14 and 15 providing extremely high selectivity (>300-fold) over CYP11B1. Interestingly, cyclopropyl derivative $\mathbf{1 6}$ had a falloff in CYP11B2 potency. Polarity at C4 was well tolerated with nitrile 17, achieving high selectivity and amine 18 providing excellent CYP11B2 inhibition, albeit with more potent CYP11B1 inhibition than seen with previous pyrimidinebased inhibitors (18 vs 12-17). N,N-Disubstitution (19) was also well tolerated as was elongation of the alkylamine (20). The stability of select compounds was evaluated in cynomolgus monkey liver microsomes, which revealed the alkyl substituted compounds $(13,14$, and 15) had moderate stability while the aminoalkyl compounds (19 and 20) were rapidly metabolized.

Given the overall profile of the simple alkyl-substituted pyrimidines, exploration of fluoroalkyl derivatives was undertaken (Table 3). Di- and trifluoromethyl analogues 21 and 22 potently inhibited CYP11B2 with excellent selectivity over CYP11B1. 1,1-Difluoroethyl derivative 23 had a modest decrease in CYP11B2 potency leading to slightly diminished selectivity. Analogues 21 and 22 both showed excellent in vitro stability. Examination of the thermodynamic aqueous solubility of trifluoromethyl derivative $\mathbf{2 2}$ revealed moderate solubility (9 $\mu \mathrm{M})$ in a $\mathrm{pH} 7.4$ buffer.

To improve upon the solubility of the pyrimidine 22, modifications to the benzimidazole core were explored. ${ }^{16} 6$ Cyanobenzimidazole and 6-cyano-7-azabenzimidazole cores with the 4-di- and trifluoromethyl pyrimidine MBGs were prepared (Table 4). All compounds were potent CYP11B2 inhibitors with analogues 24, 25, and 27 affording over 200fold selectivity and moderate (25) to excellent in vitro stability (24, 26, and 27). Gratifyingly, 6-cyano-7-azabenzimidazole 27 
Table 2. Evaluation of C4-Pyrimidine SAR

\begin{tabular}{|c|c|c|c|c|c|}
\hline cmpd & $\mathrm{R}$ & CYP11B2 $\mathrm{IC}_{50}(\mu \mathrm{M})$ & 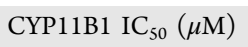 & $S^{a}$ & microsome stability $^{b}$ \\
\hline 12 & $-\mathrm{H}$ & 0.022 & 2.267 & 103 & 98 \\
\hline 13 & $-\mathrm{Me}$ & 0.017 & 4.157 & 244 & 72 \\
\hline 14 & $-\mathrm{Et}$ & 0.008 & 3.330 & 416 & 78 \\
\hline 15 & $-i \operatorname{Pr}$ & 0.005 & 1.907 & 381 & 80 \\
\hline 16 & -cyclopropyl & 0.172 & $>10.0$ & $>58$ & \\
\hline 17 & $-\mathrm{CN}$ & 0.013 & 4.987 & 383 & 85 \\
\hline 18 & -NHMe & $<0.002$ & 0.390 & $>195$ & \\
\hline 19 & $-\mathrm{N}\left(\mathrm{CH}_{3}\right)_{2}$ & 0.029 & 4.629 & 159 & 6 \\
\hline 20 & $-\mathrm{NH}\left(\mathrm{CH}_{2}\right)_{2} \mathrm{CF}_{3}$ & 0.003 & 0.378 & 126 & 55 \\
\hline
\end{tabular}

${ }^{a}$ S, Selectivity $\left(\mathrm{CYP} 11 \mathrm{~B} 1 \mathrm{IC}_{50} / \mathrm{CYP} 11 \mathrm{~B} 2 \mathrm{IC}_{50}\right) .{ }^{b}$ Percentage of parent compound remaining after a 65 min incubation in cynomolgus monkey liver microsomes.

Table 3. In Vitro Evaluation of 4-Fluoroalkylpyrimidines<smiles>[R]c1ncncc1-c1nc2cc(F)c(F)cc2n1C1CC1</smiles>

\begin{tabular}{clcccc} 
cmpd & \multicolumn{1}{c}{$\mathrm{R}$} & $\begin{array}{c}\mathrm{CYP} 11 \mathrm{~B} 2 \\
\mathrm{IC}_{50}(\mu \mathrm{M})\end{array}$ & $\begin{array}{c}\mathrm{CYP} 11 \mathrm{~B} 1 \\
\mathrm{IC}_{50}(\mu \mathrm{M})\end{array}$ & $S^{a}$ & $\begin{array}{c}\text { microsome } \\
\text { stability }^{b}\end{array}$ \\
$\mathbf{2 1}$ & $-\mathrm{CF}_{2} \mathrm{H}$ & 0.018 & 4.325 & 240 & 106 \\
$\mathbf{2 2}$ & $-\mathrm{CF}_{3}$ & 0.025 & 4.771 & 190 & 99 \\
$\mathbf{2 3}$ & $-\mathrm{CF}_{2} \mathrm{CH}_{3}$ & 0.050 & 5.723 & 114 &
\end{tabular}

${ }^{a} S$, selectivity (CYP11B1 $\left.\mathrm{IC}_{50} / \mathrm{CYP} 11 \mathrm{~B} 2 \quad \mathrm{IC}_{50}\right) .{ }^{b}$ Percentage of compound remaining after a $65 \mathrm{~min}$ incubation in cynomolgus monkey liver microsomes.

Table 4. In Vitro Evaluation of 6-Cyanobenzimidazole and 6-Cyano-7-azabenzimidazole Scaffolds<smiles>[R]c1ncncc1-c1nc2ccc(C#N)cc2n1C1CC1</smiles>

\begin{tabular}{clcccc} 
cmpd & \multicolumn{1}{c}{$\mathrm{R}, \mathrm{X}$} & $\begin{array}{c}\mathrm{CYP11B2} \\
\mathrm{IC}_{50}(\mu \mathrm{M})\end{array}$ & $\begin{array}{c}\mathrm{CYP}_{50}(\mu \mathrm{B} 1 \\
\mathrm{IC}_{50}(\mu \mathrm{M})\end{array}$ & $S^{a}$ & $\begin{array}{c}\text { microsome } \\
\text { stability }^{b}\end{array}$ \\
\hline 24 & $-\mathrm{CF}_{2} \mathrm{H}, \mathrm{CH}$ & 0.009 & 2.035 & 226 & 84 \\
25 & $-\mathrm{CF}_{3}, \mathrm{CH}$ & 0.016 & 4.171 & 261 & 62 \\
26 & $-\mathrm{CF}_{2} \mathrm{H}, \mathrm{N}$ & 0.029 & 3.926 & 135 & 101 \\
$\mathbf{2 7}$ & $-\mathrm{CF}_{3}, \mathrm{~N}$ & 0.024 & 4.809 & 200 & 87
\end{tabular}

${ }^{a} S$, selectivity (CYP11B1 IC $\left.50 / \mathrm{CYP} 11 \mathrm{~B} 2 \quad \mathrm{IC}_{50}\right) . \quad{ }^{b}$ Percentage of compound remaining after a $65 \mathrm{~min}$ incubation in cynomolgus monkey liver microsomes.

showed a 17 -fold improvement $(153 \mu \mathrm{M})$ in aqueous solubility relative to pyrimidine $\mathbf{2 2}$.

With multiple compounds meeting the desired profile for selectivity and stability, the pharmacokinetic properties of select compounds in cynomolgus monkeys were examined. Utilizing male monkeys, systemic drug concentrations from the plasma were measured following oral dosing at $1 \mathrm{mg} / \mathrm{kg}$ out to 24 or $72 \mathrm{~h}$ with data summarized in Table 5. 3-Methyl derivative 13 was rapidly absorbed $\left(T_{\max }=0.5 \mathrm{~h}\right)$ with a robust $C_{\max }(1.26 \mu \mathrm{M})$ but shorter than desired half-life. In contrast, di- and trifluoromethyl derivatives $\mathbf{2 1}$ and $\mathbf{2 2}$ provided more
Table 5. Pharmacokinetic Parameters of Pyrimidine-Based CYP11B2 Inhibitors ${ }^{a}$

$\begin{array}{ccccc}\text { cmpd } & C_{\max }(\mu \mathrm{M}) & T_{\max }(\mathrm{h}) & \mathrm{AUC}_{0-24}(\mu \mathrm{M} \mathrm{h}) & t_{1 / 2}(\mathrm{~h}) \\ \mathbf{1 3} & 1.26 & 0.5 & 3.0 & 4.2 \\ \mathbf{2 1} & 0.62 & 1.0 & 8.8 & 15.3 \\ \mathbf{2 2} & 0.41 & 2.0 & 7.8 & 30.0 \\ \mathbf{2 4} & 1.00 & 2.0 & 8.3 & 5.4 \\ \mathbf{2 6} & 2.88 & 2.0 & 32.2 & 7.5 \\ \mathbf{2 7} & 1.68 & 0.5 & 10.1 & 5.3 \\ { }^{a} \text { Dosing vehicle: } 0.5 \% \text { CMC, } 0.1 \% \text { Tween-80, 1\% DMSO. }\end{array}$

moderate $C_{\max }$ values, which are well below the concentrations required to effectively inhibit CYP11B1 but well above the levels expected for robust CYP11B2 inhibition based on the compounds' in vitro profiles. Additionally, both compounds had excellent half-lives which would be expected to provide once a day (qd) dosing in humans, thus affording an ideal PK profile for durable inhibition of aldosterone synthesis. While the pharmacokinetic profiles of compounds 24, 26, and 27 were acceptable in terms of drug exposures and half-lives, compound $\mathbf{2 2}$ was ultimately chosen for further evaluation given its superior pharmacokinetic profile. Evaluation of protein binding for compound $\mathbf{2 2}$ in cynomolgus monkey plasma revealed an unbound fraction of $16 \%$, thus affording robust free drug concentrations for efficacy studies.

Prior to evaluation of in vivo efficacy, compound 22 was profiled against the rodent and cynomolgus monkey CYP11B1 and CYP11B2 enzymes. ${ }^{24}$ As seen with previous inhibitors, the pyrimidine-based CYP11B2 compounds suffered a significant loss of potency against the rodent ortholog enzymes (data not shown). ${ }^{12,16}$ Compound $\mathbf{2 2}$ potently inhibited monkey CYP11B2 with an $\mathrm{IC}_{50}$ value of $13 \mathrm{nM}$ and afforded excellent selectivity against monkey CYP11B1, with an $\mathrm{IC}_{50}$ of $8.850 \mu \mathrm{M}$ leading to 702-fold selectivity. Against a panel of steroid and drug-metabolizing cytochrome enzymes, compound 22 was over 1000 -fold selective against the majority of the panel (CYP17, CYP2D6, CYP2C9, CYP2C19, CYP3A4) with a $\mathrm{CYP}_{19} \mathrm{IC}_{50}$ of $6.445 \mu \mathrm{M}$, providing a 250 -fold window relative to inhibition of human CYP11B2. With the desired potency and selectivity profiles achieved along with excellent pharmacokinetics, compound $\mathbf{2 2}$ was selected for evaluation in in vivo efficacy studies. 
In vivo aldosterone lowering was assessed in an ACTHinduced steroidogenesis model in conscious male cynomolgus monkeys with concurrent measurement of cortisol and steroid precursors (DOC and 11-deoxycortisol). ${ }^{25}$ In the study, control steroid responses were measured on day 1 with vehicle treatment followed by ACTH challenge to elicit adrenal steroidogenesis. This protocol was repeated after a 7-day recovery period with compound 22 administered orally at 0.2 , 1 , and $3 \mathrm{mg} / \mathrm{kg} 10 \mathrm{~min}$ prior to ACTH administration. Area under the curve (AUC) calculations were made from the plasma steroid concentrations and, using each animal's steroid AUC levels on day 1 as its own control, changes in day 8 steroid levels were determined. For comparative purposes, the nonselective inhibitor LCI699 (CYP11B2 IC $_{50}=0.7 \mathrm{nM}$; CYP11B1 $\mathrm{IC}_{50}=2.5 \mathrm{nM}$; selectivity $\left.=3.5\right)$ was also evaluated in the model. ${ }^{10}$

Plasma aldosterone AUCs were dose-dependently reduced by 68,88 , and $93 \%$ at the $0.2,1.0$, and $3.0 \mathrm{mg} / \mathrm{kg}$ doses of compound 22, respectively, with no significant impact on plasma cortisol levels (Figure 2). In addition, no change in the

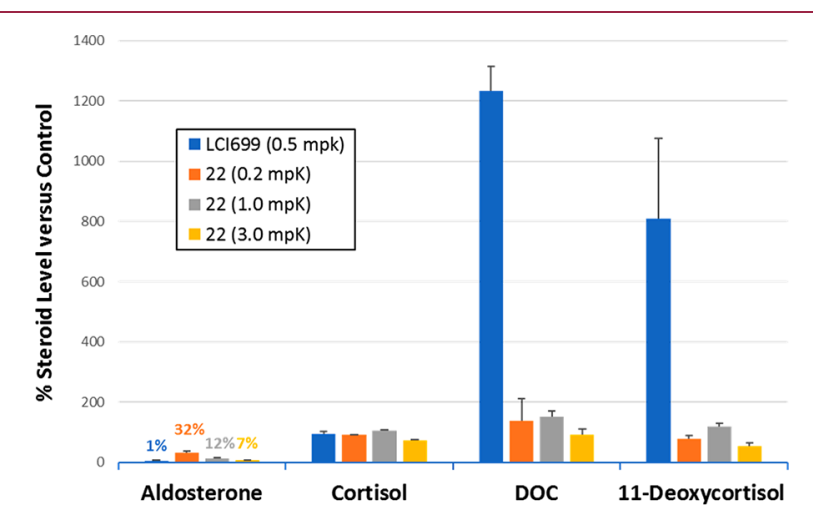

Figure 2. Effects of compound 22 and LCI699 on plasma steroid response to ACTH administration in cynomolgus monkeys.

levels of substrate steroids DOC and 11-deoxycortisol were realized with compound 22, thus providing the desired profile for selective CYP11B2 inhibition. In contrast, LCI699 (0.5 $\mathrm{mg} / \mathrm{kg}$ ) treatment caused a dramatic buildup of DOC (1 232\%) and 11-deoxycortisol (810\%), highlighting the effect of nonselective CYP11B inhibition on the steroid precursor concentrations and consistent with preceding clinical and preclinical studies. $^{25-27}$ This study demonstrates that selective CYP11B2 inhibition with pyrimidine 22 affords selective in vivo aldosterone lowering without significant impact on cortisol or precursor steroid levels.

The development of a series of pyrimidine-based CYP11B2 inhibitors is described. Modulation of the $\mathrm{C} 4$ position of the pyrimidine ring allowed for the identification of highly selective CYP11B2 inhibitor 22 which embodies an optimal PK profile for robust in vivo CYP11B2 inhibition. A dose-responsive, selective inhibition of aldosterone synthesis without effects on cortisol or steroid precursor levels, a deficit seen with LCI699, was realized with compound $\mathbf{2 2}$ in a nonhuman primate model of adrenal steroidogenesis. Further studies examining selective aldosterone lowering and blood pressure effects with selective CYP11B2 inhibitors will be reported in due course.

\section{ASSOCIATED CONTENT}

\section{S Supporting Information}

The Supporting Information is available free of charge on the ACS Publications website at DOI: 10.1021/acsmedchemlett.9b00152.

Assay protocols, experimental procedures, and analytical data for compounds 13, 22, and 27 (PDF)

\section{AUTHOR INFORMATION}

\section{Corresponding Author}

*E-mail: sparkss56@yahoo.com.

ORCID

Steven M. Sparks: 0000-0002-3869-3389

\section{Author Contributions}

All authors have given approval to the final version of the manuscript.

\section{Notes}

The authors declare no competing financial interest.

\section{ABBREVIATIONS}

$\mathrm{ACTH}$, adrenocorticotropic hormone; AUC, area under the curve; DOC, 11-deoxycorticosterone; MBG, metal binding group; mpk, mg per kg; MR, mineralocorticoid receptor.

\section{REFERENCES}

(1) Brown, N. J. Contribution of aldosterone to cardiovascular and renal inflammation and fibrosis. Nat. Rev. Nephrol. 2013, 9, 459-469.

(2) Sowers, J. R.; Whaley-Connell, A.; Epstein, M. Narrative review: the emerging clinical implications of the role of aldosterone in the metabolic syndrome and resistant hypertension. Ann. Intern. Med. 2009, 150, 776-783.

(3) Luther, J. M. Effects of aldosterone on insulin sensitivity and secretion. Steroids 2014, 91, 54-60.

(4) Deinum, J.; Riksen, N. P.; Lenders, J. W. M. Pharmacological treatment of aldosterone excess. Pharmacol. Ther. 2015, 154, 120133.

(5) Del Vecchio, L.; Procaccio, M.; Vigano, S.; Cusi, D. Mechanisms of disease: the role of aldosterone in kidney damage and clinical benefits of its blockade. Nat. Clin. Pract. Nephrol. 2007, 3, 42-49.

(6) Hundemer, G. L.; Curhan, G. C.; Yozamp, N.; Wang, M.; Vaidya, A. Renal Outcomes in Medically and Surgically Treated Primary Aldosteronism. Hypertension 2018, 72, 658-666.

(7) Hundemer, G. L.; Curhan, G. C.; Yozamp, N.; Wang, M.; Vaidya, A. Cardiometabolic outcomes and mortality in medically treated primary aldosteronism: a retrospective cohort study. Lancet Diabetes Endocrinol. 2018, 6, 51-59.

(8) Bernhardt, R. The potential of targeting CYP11B. Expert Opin. Ther. Targets 2016, 20, 923-934.

(9) Cerny, M. A. Progress Towards Clinically Useful Aldosterone Synthase Inhibitors. Curr. Top. Med. Chem. (Sharjah, United Arab Emirates) 2013, 13, 1385-1401.

(10) Meredith, E. L.; Ksander, G.; Monovich, L. G.; Papillon, J. P. N.; Liu, Q.; Miranda, K.; Morris, P.; Rao, C.; Burgis, R.; Capparelli, M.; Hu, Q.-Y.; Singh, A.; Rigel, D. F.; Jeng, A. Y.; Beil, M.; Fu, F.; Hu, C.-W.; LaSala, D. Discovery and in Vivo Evaluation of Potent Dual CYP11B2 (Aldosterone Synthase) and CYP11B1 Inhibitors. ACS Med. Chem. Lett. 2013, 4, 1203-1207.

(11) Schumacher, C. D.; Steele, R. E.; Brunner, H. R. Aldosterone synthase inhibition for the treatment of hypertension and the derived mechanistic requirements for a new therapeutic strategy. J. Hypertens. 2013, 31, 2085-2093.

(12) Hoyt, S. B.; Park, M. K.; London, C.; Xiong, Y.; Tata, J.; Bennett, D. J.; Cooke, A.; Cai, J.; Carswell, E.; Robinson, J.; MacLean, J.; Brown, L.; Belshaw, S.; Clarkson, T. R.; Liu, K.; Liang, G. B.; Struthers, M.; Cully, D.; Wisniewski, T.; Ren, N.; Bopp, C.; Sok, A.; 
Cai, T. Q.; Stribling, S.; Pai, L. Y.; Ma, X.; Metzger, J.; Verras, A.; McMasters, D.; Chen, Q.; Tung, E.; Tang, W.; Salituro, G.; Buist, N.; Kuethe, J.; Rivera, N.; Clemas, J.; Zhou, G.; Gibson, J.; Maxwell, C. A.; Lassman, M.; McLaughlin, T.; Castro-Perez, J.; Szeto, D.; Forrest, G.; Hajdu, R.; Rosenbach, M.; Ali, A. Discovery of Benzimidazole CYP11B2 Inhibitors with in Vivo Activity in Rhesus Monkeys. ACS Med. Chem. Lett. 2015, 6, 573-578.

(13) Weldon, S. M.; Cerny, M. A.; Gueneva-Boucheva, K.; Cogan, D.; Guo, X.; Moss, N.; Parmentier, J.-H.; Richman, J. R.; Reinhart, G. A.; Brown, N. F. Selectivity of BI 689648, a novel, highly selective aldosterone synthase inhibitor: comparison with FAD286 and LCI699 in nonhuman primates. J. Pharmacol. Exp. Ther. 2016, 359, 142-150.

(14) Sakakibara, R.; Sasaki, W.; Onda, Y.; Yamaguchi, M.; Ushirogochi, H.; Hiraga, Y.; Sato, K.; Nishio, M.; Egi, Y.; Takedomi, K.; Shimizu, H.; Ohbora, T.; Akahoshi, F. Discovery of Novel Pyrazole-Based Selective Aldosterone Synthase (CYP11B2) Inhibitors: A New Template to Coordinate the Heme-Iron Motif of CYP11B2. J. Med. Chem. 2018, 61, 5594-5608.

(15) Hoyt, S. B.; Petrilli, W.; London, C.; Liang, G.-B.; Tata, J.; Hu, Q.; Yin, L.; van Koppen, C. J.; Hartmann, R. W.; Struthers, M.; Wisniewski, T.; Ren, N.; Bopp, C.; Sok, A.; Cai, T.-Q.; Stribling, S.; Pai, L.-Y.; Ma, X.; Metzger, J.; Verras, A.; McMasters, D.; Chen, Q.; Tung, E.; Tang, W.; Salituro, G.; Buist, N.; Clemas, J.; Zhou, G.; Gibson, J.; Maxwell, C. A.; Lassman, M.; McLaughlin, T.; CastroPerez, J.; Szeto, D.; Forrest, G.; Hajdu, R.; Rosenbach, M.; Xiong, Y. Discovery of Triazole CYP11B2 Inhibitors with in Vivo Activity in Rhesus Monkeys. ACS Med. Chem. Lett. 2015, 6, 861-865.

(16) Papillon, J. P. N.; Lou, C.; Singh, A. K.; Adams, C. M.; Ksander, G. M.; Beil, M. E.; Chen, W.; Leung-Chu, J.; Fu, F.; Gan, L.; Hu, C.W.; Jeng, A. Y.; LaSala, D.; Liang, G.; Rigel, D. F.; Russell, K. S.; Vest, J. A.; Watson, C. Discovery of N-[5-(6-Chloro-3-cyano-1-methyl-1Hindol-2-yl)-pyridin-3-ylmethyl]-ethanesulfonamide, a Cortisol-Sparing CYP11B2 Inhibitor that Lowers Aldosterone in Human Subjects. J. Med. Chem. 2015, 58, 9382-9394.

(17) Bogman, K.; Schwab, D.; Delporte, M. L.; Palermo, G.; Amrein, K.; Mohr, S.; De Vera Mudry, M. C.; Brown, M. J.; Ferber, P. Preclinical and Early Clinical Profile of a Highly Selective and Potent Oral Inhibitor of Aldosterone Synthase (CYP11B2). Hypertension 2017, 69, 189-196.

(18) Hoekstra, W. J.; Garvey, E. P.; Moore, W. R.; Rafferty, S. W.; Yates, C. M.; Schotzinger, R. J. Design and optimization of highlyselective fungal CYP51 inhibitors. Bioorg. Med. Chem. Lett. 2014, 24, $3455-3458$.

(19) Rafferty, S. W.; Eisner, J. R.; Moore, W. R.; Schotzinger, R. J.; Hoekstra, W. J. Highly-selective 4-(1,2,3-triazole)-based P450c17a 17,20-lyase inhibitors. Bioorg. Med. Chem. Lett. 2014, 24, 2444-2447.

(20) Leach, A. G.; Kidley, N. J. Quantitatively Interpreted Enhanced Inhibition of Cytochrome P450s by Heteroaromatic Rings Containing Nitrogen. J. Chem. Inf. Model. 2011, 51, 1048-1063.

(21) For synthetic routes to compounds 13, 22, and 27 and detailed experimental protocols, see the Supporting Information.

(22) LaSala, D.; Shibanaka, Y.; Jeng, A. Y. Coexpression of CYP11B2 or CYP11B1 with adrenodoxin and adrenodoxin reductase for assessing the potency and selectivity of aldosterone synthase inhibitors. Anal. Biochem. 2009, 394, 56-61.

(23) For the first report of pyrimidine-based inhibitors, see Ulmschneider, S.; Mueller-Vieira, U.; Klein, C. D.; Antes, I.; Lengauer, T.; Hartmann, R. W. Synthesis and Evaluation of (Pyridylmethylene)tetrahydronaphthalenes/-indanes and Structurally Modified Derivatives: Potent and Selective Inhibitors of Aldosterone Synthase. J. Med. Chem. 2005, 48, 1563-1575.

(24) Cerny, M. A.; Csengery, A.; Schmenk, J.; Frederick, K. Development of CYP11B1 and CYP11B2 assays utilizing homogenates of adrenal glands: Utility of monkey as a surrogate for human. $J$. Steroid Biochem. Mol. Biol. 2015, 154, 197-205.

(25) Menard, J.; Rigel, D. F.; Watson, C.; Jeng, A. Y.; Fu, F.; Beil, M.; Liu, J.; Chen, W.; Hu, C.-W.; Chu, J. L.; LaSala, D.; Liang, G.; Rebello, S.; Zhang, Y.; Dole, W. P. Aldosterone synthase inhibition: cardiorenal protection in animal disease models and translation of hormonal effects to human subjects. J. Transl. Med. 2014, 12, 340.

(26) Andersen, K.; Hartman, D.; Peppard, T.; Hermann, D.; Van Ess, P.; Lefkowitz, M.; Trapani, A. The effects of aldosterone synthase inhibition on aldosterone and cortisol in patients with hypertension: a phase II, randomized, double-blind, placebo-controlled, multicenter study. J. Clin. Hypertens. (Hoboken, NJ, U. S.) 2012, 14, 580-587.

(27) Cai, T.-Q.; Stribling, S.; Tong, X.; Xu, L.; Wisniewski, T.; Fontenot, J. A.; Struthers, M.; Akinsanya, K. O. Rhesus monkey model for concurrent analyses of in vivo selectivity, pharmacokinetics and pharmacodynamics of aldosterone synthase inhibitors. J. Pharmacol. Toxicol. Methods 2015, 71, 137-146. 